SLAC-PUB-8114

April 1999

\title{
Tests of Low Scale Gravity via Gauge Boson Pair Production in $\gamma \gamma$ Collisions *
}

\author{
Thomas G. Rizzo \\ Stanford Linear Accelerator Center \\ Stanford University \\ Stanford CA 94309, USA
}

\begin{abstract}
Arkani-Hamed, Dimopoulos and Dvali have recently proposed that gravity may become strong at energies near $1 \mathrm{TeV}$ thus removing the hierarchy problem. This scenario can be tested in several ways at present and future colliders. In this paper we examine the exchange of towers of Kaluza-Klein gravitons and their influence on the production of pairs of massive gauge bosons in $\gamma \gamma$ collisions. These tower exchanges are shown to lead to a new dimension- 8 operator that can significant alter the Standard Model expectations for these processes. The role of polarization for both the initial state photons and the final state gauge bosons in improving sensitivity to graviton exchange is emphasized. We find that the discovery reach for graviton tower exchange in the $\gamma \gamma \rightarrow W^{+} W^{-}$channel to be significantly greater than for any other process so far examined.
\end{abstract}

${ }^{*}$ Work supported by the Department of Energy, Contract DE-AC03-76SF00515 


\section{Introduction}

Arkani-Hamed, Dimopoulos and Dvali(ADD) [1] have recently proposed a uniquely interesting solution to the hierarchy problem. ADD hypothesize the existence of $n$ additional large spatial dimensions in which gravity (and perhaps Standard Model singlet fields) can live, called 'the bulk', whereas all of the fields of the Standard Model(SM) are constrained to lie on 'the wall', which is our conventional 4-dimensional world. Gravity thus appears to us as weak in ordinary 4-dimensional space-time since we merely observe it's action on the wall. It has been shown [1] that such a scenario can emerge in string models where the effective Planck scale in the bulk is identified with the string scale. In such a theory the hierarchy is removed by postulating that the string or effective Planck scale in the bulk, $M_{s}$, is not far above the weak scale, e.g., a few TeV. Gauss' Law then provides a link between the values of $M_{s}$, the conventional Planck scale $M_{p l}$, and the size of the compactified extra dimensions, $R$,

$$
M_{p l}^{2} \sim R^{n} M_{s}^{n+2}
$$

where the constant of proportionality depends not only on the value of $n$ but upon the geometry of the compactified dimensions. If $M_{s}$ is near a TeV then $R \sim 10^{30 / n-19}$ meters; for separations between two masses less than $R$ the gravitational force law becomes $1 / r^{2+n}$. For $n=1, R \sim 10^{11}$ meters and is thus obviously excluded, but, for $n=2$ one obtains $R \sim 1 \mathrm{~mm}$, which is at the edge of the sensitivity for existing experiments[2]. For $2<n$, the value of $R$ is only further reduced and thus we may conclude that the range $2 \leq n$ is of phenomenological interest. Astrophysical arguments based on supernova cooling[3] seem to require that $M_{s}>50 \mathrm{TeV}$ for $n=2$, but allow $M_{s} \sim 1 \mathrm{TeV}$ for $n>2$ while cosmological arguments suggest[4] an even stronger constraint, $M_{s}>110 \mathrm{TeV}$, for $n=2$.

The Feynman rules for this scenario are obtained by considering a linearized theory of 
gravity in the bulk, decomposing it into the more familiar 4-dimensional states and recalling the existence of Kaluza-Klein towers for each of the conventionally massless fields. The entire set of fields in the K-K tower couples in an identical fashion to the particles of the SM. By considering the forms of the $4+n$ symmetric conserved stress-energy tensor for the various SM fields and by remembering that such fields live only on the wall one may derive all of the necessary couplings. An important result of these considerations is that only the massive spin-2 K-K towers (which couple to the 4-dimensional stress-energy tensor, $T^{\mu \nu}$ ) and spin-0 K-K towers (which couple proportional to the trace of $T^{\mu \nu}$ ) are of phenomenological relevance as all the spin-1 fields can be shown to decouple from the particles of the SM. For processes that involve massless fields at at least one vertex, as will be the case below, the contributions of the spin-0 fields can also be ignored.

The detailed phenomenology of the ADD model has begun to be explored for a wide ranging set of processes in a rapidly growing series of recent papers [5]. Given the Feynman rules as developed by Guidice, Rattazzi and Wells and by Han, Lykken and Zhang[5], it appears that the ADD scenario has two basic classes of collider tests. In the first class, type- $i$, a K-K tower of gravitons can be emitted during a decay or scattering process leading to a final state with missing energy. The rate for such processes is strongly dependent on the number of extra dimensions as well as the exact value of $M_{s}$. However, in this case the value of $M_{s}$ is directly probed. In the second class, type- $i i$, which we consider here, the exchange of a K-K graviton tower between SM or MSSM fields can lead to almost $n$-independent modifications to conventional cross sections and distributions or they can possibly lead to new interactions. The exchange of the graviton K-K tower leads to a set of effective color and flavor singlet contact interaction operator of dimension-eight with the overall scale set by the cut-off in the tower summation, $\Lambda$. Naively $\Lambda$ and $M_{s}$ should be of comparable magnitude and so one introduces a universal overall order one coefficient for these operators, 
$\lambda$ ( whose value is unknown but can be approximated by a constant which has conventionally been set to \pm 1 ) with $\Lambda$ being replaced by $M_{s}$. The fact that $\Lambda$ can be smaller than $M_{s}$ is thus particularly important when considering the case of 2 extra dimensions due to the rather strong astrophysical and cosmological constraints that then apply. We note that $\lambda$ can in principle have either sign since the unknown physics above the cut-off can make an additional universal contribution to the coefficient of the relevant amplitude of indeterminate sign. Given the kinematic structure of these operators the modifications in the relevant cross sections and distributions can be directly calculated.

In what follows we will consider the production of massive gauge boson pairs in $\gamma \gamma$ collisions via the exchange of a K-K tower of gravitons (the process $\gamma \gamma \rightarrow \gamma \gamma$ having been considered[5] elsewhere). Such reactions can be examined in detail at future linear colliders via the Compton back-scattering of laser photons off of high energy colliding $e^{+} e^{-}$or $e^{-} e^{-}$ beams[6]. As we note below, the role of polarization, in both the initial state as well as

the final state, is crucial in separating the graviton signal from the SM background and extending the search reach. We then will demonstrate that the discovery reach for graviton tower exchange in the $W W$ channel is greater than any other process so far examined. The corresponding reach in the $Z Z$ case will be shown to be rather modest and comparable to that found for the $\gamma \gamma \rightarrow \gamma \gamma$ process.

\section{$2 \quad \gamma \gamma \rightarrow V V$ via Graviton Exchange}

$\gamma \gamma$ collisions offer a unique and distinct window on the possibility of new physics in a particularly clean environment. At tree level the cross section for particle pair production, if it is allowed by the gauge symmetries, depends only gauge couplings. Unlike gauge boson pair production in $e^{+} e^{-}$collisions, however, $P, C$, plus the Bose symmetry of the initial state pho- 
tons forbids the existence of non-zero values, at the tree level, for either forward-backward angular asymmetries or left-right forward-backward polarization asymmetries. These were both found to be powerful tools in probing for K-K graviton tower exchanges in the $e^{+} e^{-}$ initiated channels[5]. In the case of $\gamma \gamma$ collisions our remaining tools are the angular distributions of the produced pairs of vector bosons, their resulting states of polarization, and the cross section's sensitivity to the polarization of the initial state photons.

The exchange of a tower of K-K gravitons leads to a new tree level contribution to the process $\gamma\left(k_{1}, \epsilon_{1}\right) \gamma\left(k_{2}, \epsilon_{2}\right) \rightarrow V\left(p_{1}, V_{1}\right) V\left(p_{2}, V_{2}\right)$, where $k_{1,2}$ are incoming and $p_{1,2}$ are outgoing momenta and $\epsilon_{1,2}\left(V_{1,2}\right)$ represent the polarization vectors for the photons(vector bosons), respectively. Following the Feynman rules of either Guidice, Rattazzi and Wells or Han, Lykken and Zhang[5] we can immediately write down the relevant matrix element which has the form of a dimension-8 operator:

$$
\mathcal{M}_{\text {grav }}=\frac{8 \lambda K}{M_{s}^{4}}\left[M_{1}+M_{2}+M_{3}+M_{4}+M_{5}\right]
$$

where

$$
\begin{aligned}
M_{1} & =\frac{s^{2}}{2}\left[\epsilon_{1} \cdot V_{1}^{*} \epsilon_{2} \cdot V_{2}^{*}+\epsilon_{1} \cdot V_{2}^{*} \epsilon_{2} \cdot V_{1}^{*}\right]+s \epsilon_{1} \cdot \epsilon_{2}\left[k_{1} \cdot V_{1}^{*} k_{2} \cdot V_{2}^{*}+k_{1} \cdot V_{2}^{*} k_{2} \cdot V_{1}^{*}\right. \\
& \left.-k_{1} \cdot k_{2} V_{1}^{*} \cdot V_{2}^{*}\right] \\
M_{2} & =s V_{1}^{*} \cdot V_{2}^{*}\left[p_{1} \cdot \epsilon_{1} p_{2} \cdot \epsilon_{2}+p_{1} \cdot \epsilon_{2} p_{2} \cdot \epsilon_{1}\right]+2 \epsilon_{1} \cdot \epsilon_{2} V_{1}^{*} \cdot V_{2}^{*}\left[p_{1} \cdot k_{1} p_{2} \cdot k_{2}+p_{1} \cdot k_{2} p_{2} \cdot k_{1}\right. \\
& \left.-p_{1} \cdot p_{2} k_{1} \cdot k_{2}\right] \\
M_{3} & =-s p_{1} \cdot V_{2}^{*}\left[\epsilon_{1} \cdot V_{1}^{*} \epsilon_{2} \cdot p_{2}+\epsilon_{2} \cdot V_{1}^{*} \epsilon_{1} \cdot p_{2}\right]-s p_{2} \cdot V_{1}^{*}\left[\epsilon_{1} \cdot V_{2}^{*} \epsilon_{2} \cdot p_{1}+\epsilon_{2} \cdot V_{2}^{*} \epsilon_{1} \cdot p_{1}\right] \\
M_{4} & =-2 p_{1} \cdot V_{2}^{*} \epsilon_{1} \cdot \epsilon_{2}\left[k_{1} \cdot V_{1}^{*} k_{2} \cdot p_{2}+k_{2} \cdot V_{1}^{*} k_{1} \cdot p_{2}-p_{2} \cdot V_{1}^{*} k_{1} \cdot k_{2}\right]
\end{aligned}
$$




$$
M_{5}=-2 p_{2} \cdot V_{2}^{*} \epsilon_{1} \cdot \epsilon_{2}\left[k_{1} \cdot V_{2}^{*} k_{2} \cdot p_{1}+k_{2} \cdot V_{2}^{*} k_{1} \cdot p_{1}-p_{1} \cdot V_{2}^{*} k_{1} \cdot k_{2}\right]
$$

with $s$ being the usual Mandelstam variable. We note that in the expression above $M_{s}$ should actually be the cut-off scale used in performing the summation over the K-K tower in the $s$-channel. In principle, as discussed above, these two scales may differ by by some factor of order unity which we thus incorporate into the parameter $\lambda$. For $K=1(\pi / 2)$ we recover the normalization convention employed by Hewett(Guidice, Rattazzi and Wells)[5]; we will take $K=1$ in the numerical analysis that follows but keep the factor in our analytical expressions. We recall from the original Hewett analysis that $\lambda$ is to be treated as a parameter of order unity whose sign is undetermined and that, given the scaling relationship between $\lambda$ and $M_{s}$, experiments in the case of processes of type- $i i$ actually probe only the combination $M_{s} /|K \lambda|^{1 / 4}$. For simplicity in what follows we will numerically set $|\lambda|=1$ and employ $K=1$ but we caution the reader about this technicality and quote our sensitivity to $M_{s}$ for $\lambda= \pm 1$ as is now the standard tradition.

In the center of mass frame we imagine that the initial photons are coming in along the $z$-axis with the outgoing vector bosons making an angle $\theta$ with respect to this axis. Using $\beta^{2}=1-4 m^{2} / s, m$ being the mass of the field $V$, and recalling that the transverse initial state photons are assumed to be circularly polarized, we can describe the complete kinematics for the process in terms of the following four-vectors:

$$
\begin{aligned}
k_{1 \mu} & =\frac{\sqrt{s}}{2}(1,0,0,1) & k_{2 \mu} & =\frac{\sqrt{s}}{2}(1,0,0,-1) \\
p_{1 \mu} & =\frac{\sqrt{s}}{2}\left(1, \beta s_{\theta}, 0, \beta c_{\theta}\right) & p_{2 \mu} & =\frac{\sqrt{s}}{2}\left(1,-\beta s_{\theta}, 0,-\beta c_{\theta}\right) \\
\epsilon_{1}^{\mu} & =-\frac{1}{\sqrt{2}}\left(0, \lambda_{1}, i, 0\right) & \epsilon_{2}^{\mu} & =\frac{1}{\sqrt{2}}\left(0,-\lambda_{2}, i, 0\right) \\
V_{1 L}^{\mu *} & =\frac{\sqrt{s}}{2 m}\left(-\beta, s_{\theta}, 0, c_{\theta}\right) & V_{2 L}^{\mu *} & =-\frac{\sqrt{s}}{2 m}\left(\beta, s_{\theta}, 0, c_{\theta}\right) \\
V_{1 T}^{\mu *} & =\frac{1}{\sqrt{2}}\left(0,-\lambda_{1}^{V} c_{\theta}, i, \lambda_{1}^{V} s_{\theta}\right) & V_{2 T}^{\mu *} & =\frac{1}{\sqrt{2}}\left(0,-\lambda_{2}^{V} c_{\theta},-i, \lambda_{2}^{V} s_{\theta}\right)
\end{aligned}
$$

with $s_{\theta}=\sin \theta$ and $z=c_{\theta}=\cos \theta$. The use of covariant and contravariant indices in these 
expressions should be noted by the reader. Here the indices $T, L$ on the polarization vectors $V_{1,2}$ denote states of transverse and longitudinal polarization. The quantities $\lambda_{1,2}$ and $\lambda_{1,2}^{V}$ parameterize the two transverse polarization states of the photons and vector bosons, respectively, and take on the values \pm 1 . Given these kinematical expressions we can immediately evaluate all of the dot products appearing in the matrix element for any given choice of photon and/or $V$ polarizations. The resulting differential cross section for any particularly chosen set of helicities can then be written as

$$
\frac{d \sigma}{d z}=\frac{1}{1+\delta_{V Z}} \frac{\beta}{32 \pi s}\left|\mathcal{M}_{S M}+\mathcal{M}_{\text {grav }}\right|^{2},
$$

where $\mathcal{M}_{S M}$ symbolically represents the more conventional contribution to these matrix elements arising in the SM or the MSSM, etc. Note the Kronecker delta factor in the denominator in the case of the identical particle $Z Z$ final state.

Unfortunately $\gamma \gamma$ collisions at future linear colliders will not be as straightforward as the description above would indicate since the photons in the collision will not be either monoenergetic or in a unique state of polarization. Polarized $\gamma \gamma$ collisions may be possible at future $e^{+} e^{-}$colliders through the use of Compton backscattering of polarized low energy laser beams off of polarized high energy electrons[6]. The resulting backscattered photon distribution, $f_{\gamma}\left(x=E_{\gamma} / E_{e}\right)$, is as stated above far from monoenergetic and is cut off above $x_{\max } \simeq 0.83$ implying that the colliding photons are significantly softer than the parent lepton beam energy. As one sees, this cutoff at large $x, x_{\text {max }}$, implies that the $\gamma \gamma$ center of mass energy never exceeds $\simeq 0.83$ of the parent collider and this has resulted in a significantly degraded $M_{s}$ sensitivity for final states involving fermion or scalar pairs[5]. In addition, both the shape of the function $f_{\gamma}$ and the average helicity of the produced $\gamma$ 's are quite sensitive to the polarization state of both the initial laser $\left(P_{l}\right)$ and electron $\left(P_{e}\right)$ whose values fix the specific distribution. 
While it is anticipated that the initial laser polarization will be near $100 \%$, i.e., $\left|P_{l}\right|=1$, the electron beam polarization is expected to be be near $90 \%$, i.e., $\left|P_{e}\right|=0.9$. We will assume these values in the analysis that follows. With two photon 'beams' and the choices $P_{l}= \pm 1$ and $P_{e}= \pm 0.9$ to be made for each beam it would appear that 16 distinct polarization-dependent cross sections need to be examined. However, due to the exchange symmetry between the two photons and the fact that a simultaneous flip in the signs of all the polarizations leaves the product of the fluxes, mean helicities and the cross sections invariant, we find that there only six physically distinct initial polarization combinations[7]. In what follows we will label these possibilities by the corresponding signs of the electron and laser polarizations as $\left(P_{e 1}, P_{l 1}, P_{e 2}, P_{l 2}\right)$, For example, the configuration $(-++-)$ corresponds to $P_{e 1}=-0.9, P_{l 1}=+1, P_{e 2}=0.9$ and $P_{l 2}=-1$. Clearly some of these polarization combinations will be more sensitive to the effects of K-K towers of gravitons than will others so our analysis can be used to pick out those particular cases. Within this framework we must view the above differential cross section as that of a partonic subprocess in analogy with hadronic collisions, i.e., $d \sigma \rightarrow d \hat{\sigma}$ and we identify $s \rightarrow \hat{s}=s_{e^{+} e^{-}} x_{1} x_{2}$.

For any given choice of the initial state laser and electron polarizations, labelled by $(a, b)$ below, we can immediately write down the appropriate cross section by folding in the corresponding photon fluxes and integrating:

$$
\frac{d \sigma^{a b}}{d z}=\int d x_{1} \int d x_{2} f_{\gamma}^{a}\left(x_{1}, \xi_{1}\right) f_{\gamma}^{b}\left(x_{2}, \xi_{2}\right)\left[\frac{1+\xi_{1} \xi_{2}}{2} \frac{d \hat{\sigma}_{++}}{d z}+\frac{1-\xi_{1} \xi_{2}}{2} \frac{d \hat{\sigma}_{+-}}{d z}\right]
$$

where we explicitly note the dependence of the fluxes on the mean helicities $\xi_{1,2}$ which are known functions of energy as well as both the initial state laser and electron beam polarization. The ++ and +- labels on the subprocess cross sections indicate the appropriate values of $\lambda_{1,2}$ to chose in their evaluation. In order to obtain $\frac{d \sigma}{d z}$ the polarizations of the vector bosons in the final state must be either specified or summed over. Similarly, we can 
obtain the unpolarized cross section by averaging over the initial state photon polarizations. Given the fluxes[6] these integrals are easily evaluated numerically. The upper limit of both integrals is just $x_{\max }$. In the present case the kinematics require the photon energies to satisfy the constraint $\tau=\hat{s} / s=x_{1} x_{2} \geq 4 m^{2} / s=\tau_{\min }$ which, together with the value of $x_{\text {max }}$, then determines the lower bounds on both $x_{1,2}: x_{1}^{\min }=\tau_{\min } / x_{\max }$ and $x_{2}^{\min }=\tau_{\min } / x_{1}$.

\section{Results}

Now that we have specified all of the relevant machinery we can now turn to some results. We consider the two cases $V=W$ and $V=Z$ separately.

\section{$3.1 \quad \gamma \gamma \rightarrow W^{+} W^{-}$}

The tree-level SM helicity amplitudes for the process $\gamma \gamma \rightarrow W^{+} W^{-}$have been known for some time[8] and even the complete one-loop corrections within the SM are also known[9]. In what follows we will ignore these loop corrections but remind the reader that they must be employed in a complete analysis that also includes detector effects etc. Given these amplitudes, we can immediately calculate the relevant differential cross section combining SM and graviton tower exchanges. To be definitive we will assume $\sqrt{s}=\sqrt{s}_{e^{+} e^{-}}=1 \mathrm{TeV}$ with $M_{s}=2.5 \mathrm{TeV}$ for purposes of demonstration.

The results are shown in Fig.1 for the SM case as well as when the K-K tower is turned on with either sign of $\lambda$ for all six initial helicity combinations. In the SM case the shape of the angular distribution is easily understood by recalling that the $\gamma \gamma \rightarrow W^{+} W^{-}$ reaction takes place through $t$ - and $u$-channel $W$ exchanges as well as a $\gamma \gamma W^{+} W^{-}$four-point interaction. The $t$ - and $u$-channel exchanges thus lead to a sharply rising cross section in

both the forward and backward directions. Note that in the SM there is no dramatically 

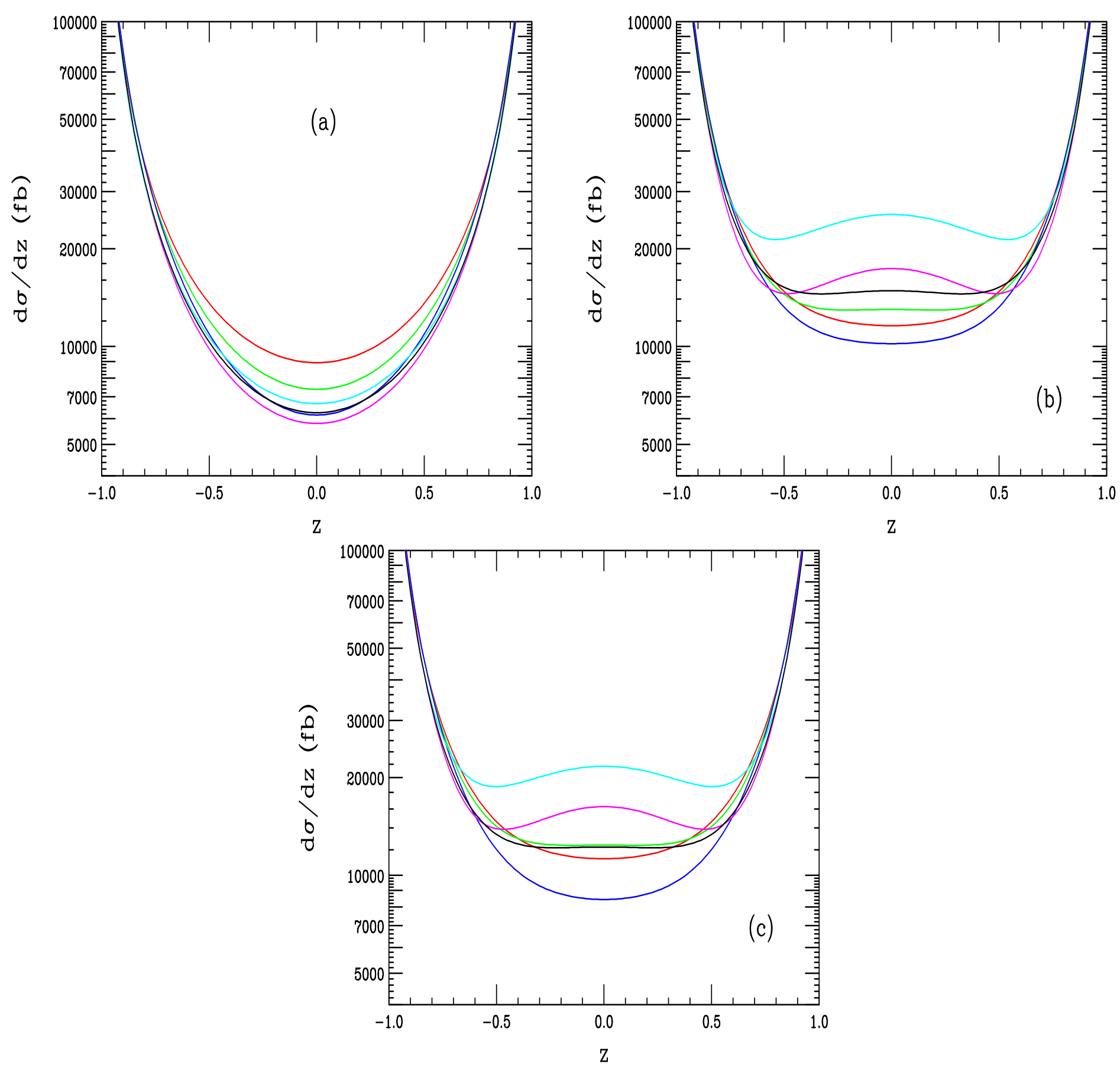

Figure 1: Differential cross section for $\gamma \gamma \rightarrow W^{+} W^{-}$at a $1 \mathrm{TeV} e^{+} e^{-}$collider for (a)the $\mathrm{SM}$ and with $M_{s}=2.5 \mathrm{TeV}$ with $(\mathrm{b}) \lambda=1$ or $(\mathrm{c}) \lambda=-1$. In (a) from top to bottom in the center of the figure the helicities are $(++++),(+++-),(-++-),(++--),(+---)$, and $(+-+-)$; in (b) they are $(-++-),(+-+-),(+++-),(+---),(++++)$, and $(++--)$; in $(\mathrm{c})$ they are $(-++-),(+-+-),(+---),(+++-),(++++)$, and $(++--)$. 
strong sensitivity to the initial state lepton and laser polarizations in this case and all of the curves have roughly the same shape. In all cases the total cross section, even after generous angular cuts, is quite enormous, of order $\sim 100 \mathrm{pb}$, providing huge statistics to look for new physics influences. When the graviton terms are turned on there are several effects. First, all of differential cross section distributions become somewhat more shallow, particularly in the case of $\lambda=1$, but there is little change in the forward and backward directions due to the dominance of the SM poles. Second, there is now a clear and distinct sensitivity to the initial state polarization selections. In some cases, particularly for the $(-++-)$ and $(+-+-)$ helicity choices, the differential cross section increases significantly for angles near $90^{\circ}$ taking on an m-like shape. This shape is, in fact, symptomatic of the spin- 2 nature of the K-K graviton tower exchange since a spin-0 exchange leads only to a flattened distribution. Given the very large statistics available with a typical integrated luminosity of $100 \mathrm{fb}^{-1}$, even after angular cuts are applied to remove the forward and backward SM poles, it is clear that the $\gamma \gamma \rightarrow W^{+} W^{-}$differential cross section is quite sensitive to $M_{s}$ especially for the two initial state helicities specified above.

In addition to a significant modification to the angular distribution, the K-K graviton tower exchange leads to another important effect through its influence on the polarizations of the two $W$ 's in the final state. In the SM, independent of the initial electron and laser polarizations, the final state $W$ 's are dominantly transversely polarized. Due to the nature of the spin-2 graviton exchange, the K-K tower leads to a final state where both $W$ 's are completely longitudinally polarized. Thus we might expect that a measurement of the $W$ polarization will probe $M_{s}$. To see this, we show in Fig.2 the polarization fractions of the two $W$ 's as a function of $M_{s}$ at a $1 \mathrm{TeV}$ collider assuming two different choices of the initial state polarizations. In the results presented in this figure, an angular cut of $|z|<0.8$ has been applied to remove the SM poles in the forward and backward directions. Here we see 

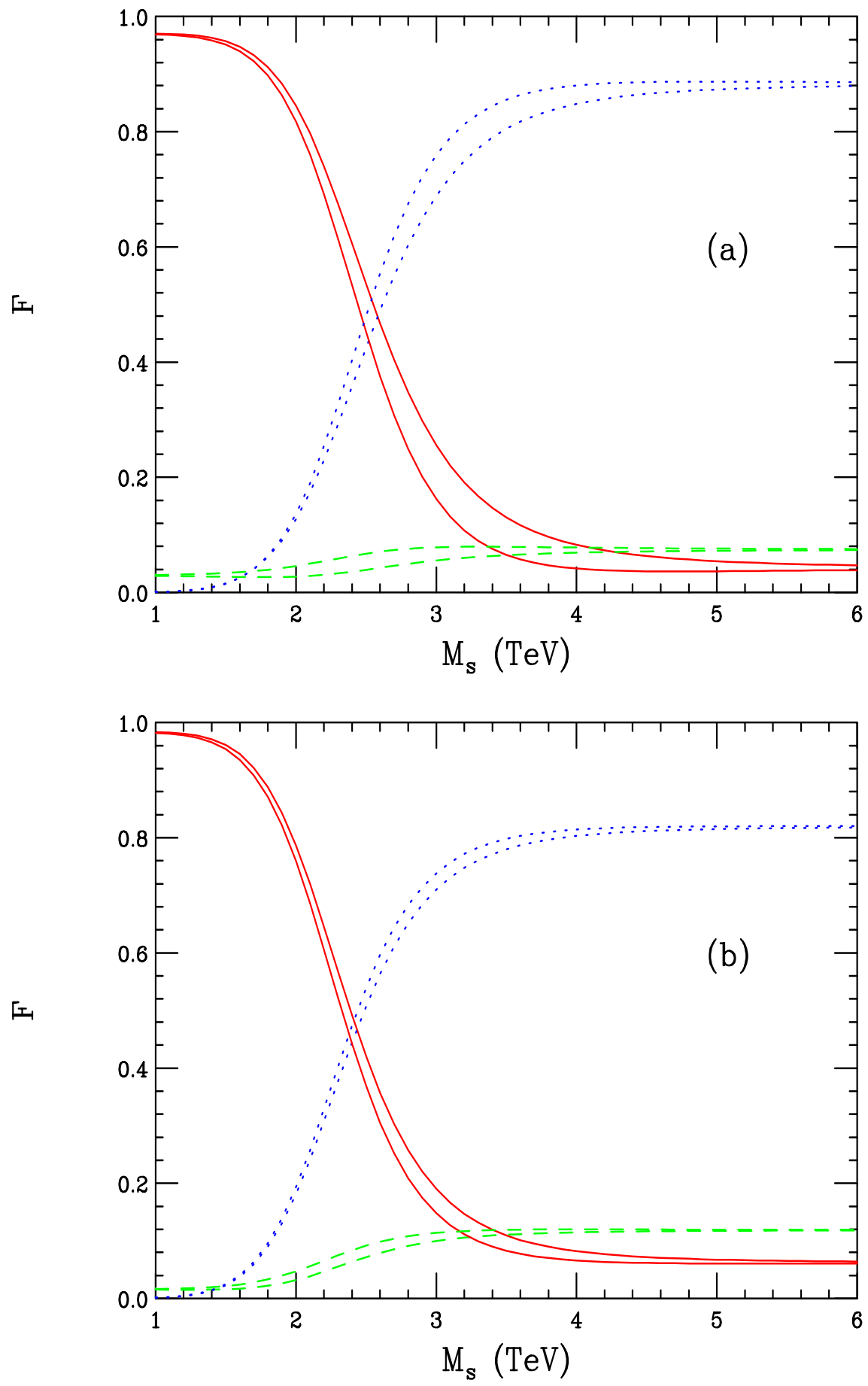

Figure 2: Fraction of LL(solid), TL+LT(dashed) and TT(dotted) $W^{+} W^{-}$final states after angular cuts for the process $\gamma \gamma \rightarrow W^{+} W^{-}$at a $1 \mathrm{TeV} e^{+} e^{-}$collider as a function of $M_{s}$ for either sign of $\lambda$. The initial state polarization in (a) is $(-++-)$ whereas in (b) it is $(+-+-)$. 
that the fraction of final states where both $W^{\prime}$ 's are longitudinal, denoted by $L L$, starts out near unity but falls significantly in the $M_{s}=2.5-3 \mathrm{TeV}$ region giving essentially the SM results above about 5.5-6 TeV. The reverse situation is observed for the case where both $W$ 's are transversely polarized, denoted by $T T$. The mixed case, denoted by the sum $T L+L T$, is also seen to grow from near zero to a modest value as $M_{s}$ increases and the SM limit is reached. Clearly a measurement of these final $W$ polarizations will allow us to probe respectable values of $M_{s}$. In order to determine the polarization fractions of the final state $W$ 's one needs to examine the correlations in the angular distributions of the fermion decay products relative to the $W$ 's original direction. If $\chi$ is the angle of one of the fermions with respect to the $W$ direction in the $W$ rest frame then transverse(longitudinal) $W$ 's lead to an angular distribution $\sim 1+(-) \cos ^{2} \chi$. Thus by measuring the correlation for both $W$ 's the relevant $L L, T T$ and $L T+T L$ fractions can be extracted. In our numerical exercise we will assume that this can be done with an efficiency of $\sim 50 \%$ for the $W W$ all hadronic final states ( $W$ mass reconstruction removing combinatoric problems) as well as for the $q \bar{q} \ell \nu$ final state with no significant backgrounds present in either case due to the very large $\gamma \gamma \rightarrow W^{+} W^{-}$ cross section.

By combining a fit to the total cross sections and angular distributions as well as the $L L$ and $L T+T L$ helicity fractions for various initial state polarization choices we are able to discern the discovery as well as the $95 \% \mathrm{CL}$ exclusion reaches for $M_{s}$. Given the rather steep behaviour of the both the SM-gravity and pure gravity terms in the cross section with $M_{s}$, i.e., $M_{s}^{-4}$ and $M_{s}^{-8}$ respectively, we do not expect these two reaches to differ significantly. In performing this analysis, in addition to the above efficiencies and angular cuts, we have assumed an overall integrated luminosity uncertainty of $1 \%$. The results of this analysis for $\lambda= \pm 1$ and the six possible initial state polarizations are displayed in Figs.3 and 4 which show both reaches as functions of the total integrated luminosity. Note both the strong 

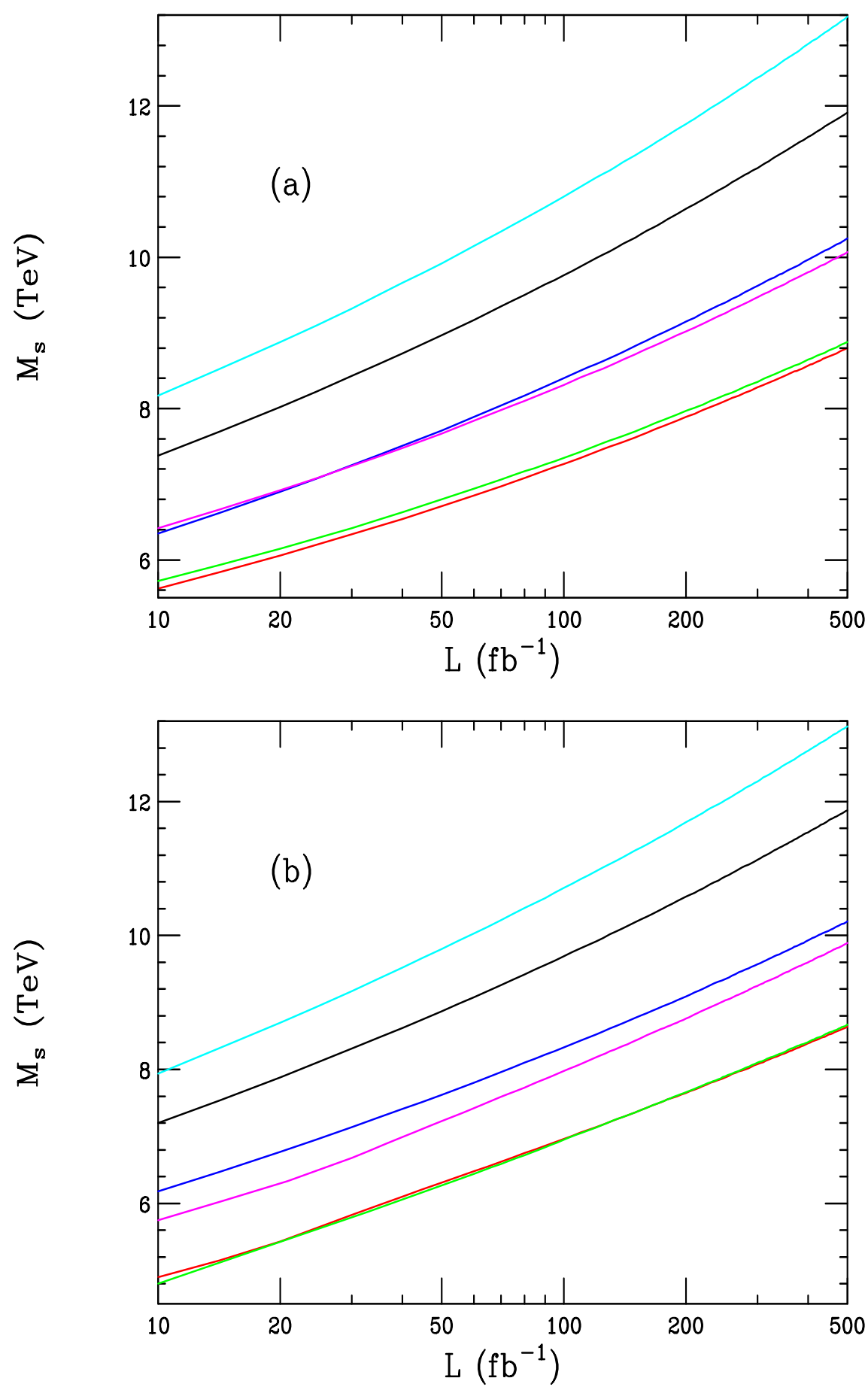

Figure 3: $M_{s}$ discovery reach from the process $\gamma \gamma \rightarrow W^{+} W^{-}$at a $1 \mathrm{TeV} e^{+} e^{-}$collider as a function of the integrated luminosity for the different initial state polarizations assuming (a) $\lambda=1$ or $(\mathrm{b}) \lambda=-1$. From top to bottom on the right hand side of the figure the polarizations are $(-++-),(+---),(++--),(+-+-),(+---)$, and $(++++)$. 
sensitivity of the two reaches to the initial electron and laser polarizations as well as the large values obtainable particularly for the $(-++-)$ choice. In this particular case with 100 $f b^{-1}$ of integrated luminosity the discovery reach is almost $11 \sqrt{s}$ for either sign of $\lambda$, which is greater than any other $\mathrm{K}-\mathrm{K}$ graviton exchange process so far examined[5]. Clearly, a more detailed analysis of these reaches, including both radiative corrections and detector effects, is more than warranted.

There are of course many more observables available that are sensitive to the presence of the K-K graviton tower exchange and which can be included in a more detailed global fit; here we only mention two of them. First, we can construct the invariant mass distribution of the two $W$ 's in the final state approximately $90 \%$ of the time and thus determine the differential cross section $d \sigma / d M_{W W}$. After imposing the $|z|<0.8$ cut we would expect that the SM result will gradually fall off with increasing $M_{W W}$. However, since the K-K graviton tower exchange contribution grows quite rapidly with increasing $\hat{s}$, a modest value of $M_{s}$ will leads to an observable event excess at large values of $M_{W W}$. This is in fact exactly what we find as shown in Fig.5 for the sample choice of the initial state polarization $(-++-)$. Clearly a fit to this just distribution alone with the large statistics available will provide an additional probe of $M_{s}$ in the range beyond $4 \mathrm{TeV}$.

Second, for the six possible initial state polarizations one can construct three independent polarization asymmetries of the form

$$
A_{p o l}=\frac{\sigma\left(P_{e 1}, P_{l 1}, P_{e 2}, P_{l 2}\right)-\sigma\left(P_{e 1}, P_{l 1},-P_{e 2},-P_{l 2}\right)}{\sigma\left(P_{e 1}, P_{l 1}, P_{e 2}, P_{l 2}\right)+\sigma\left(P_{e 1}, P_{l 1},-P_{e 2},-P_{l 2}\right)}
$$

These asymmetries can also be made angular dependent, $A_{\text {pol }}(z)$, by interpreting the cross sections in both the numerator and denominator as differential in $z$ and taking advantage of the large statistics available. Further one may separately examine asymmetries constructed 

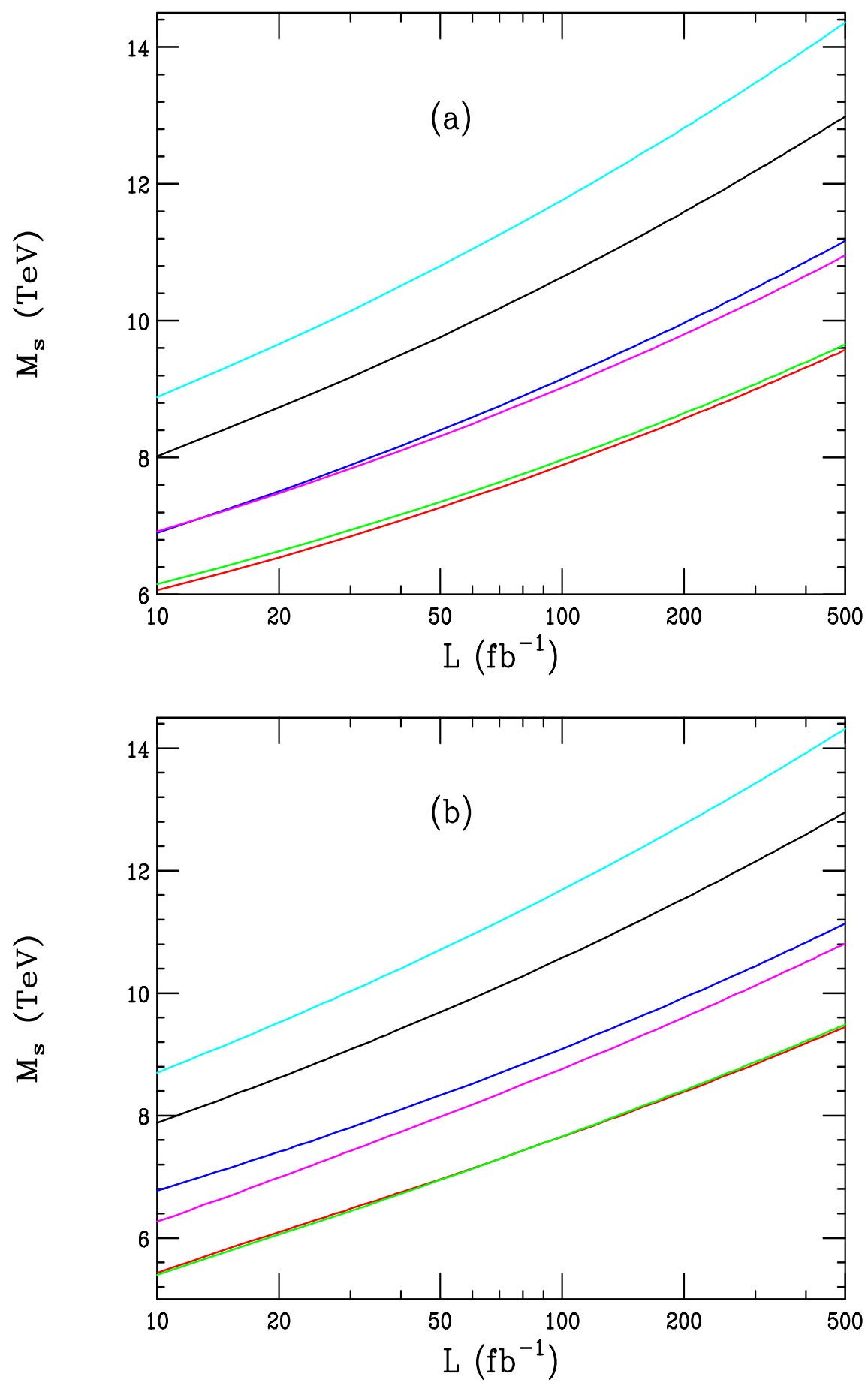

Figure 4: $95 \%$ CL exclusion reach for $M_{s}$ at a $1 \mathrm{TeV} e^{+} e^{-}$collider for the same cases as shown in the previous figure. 


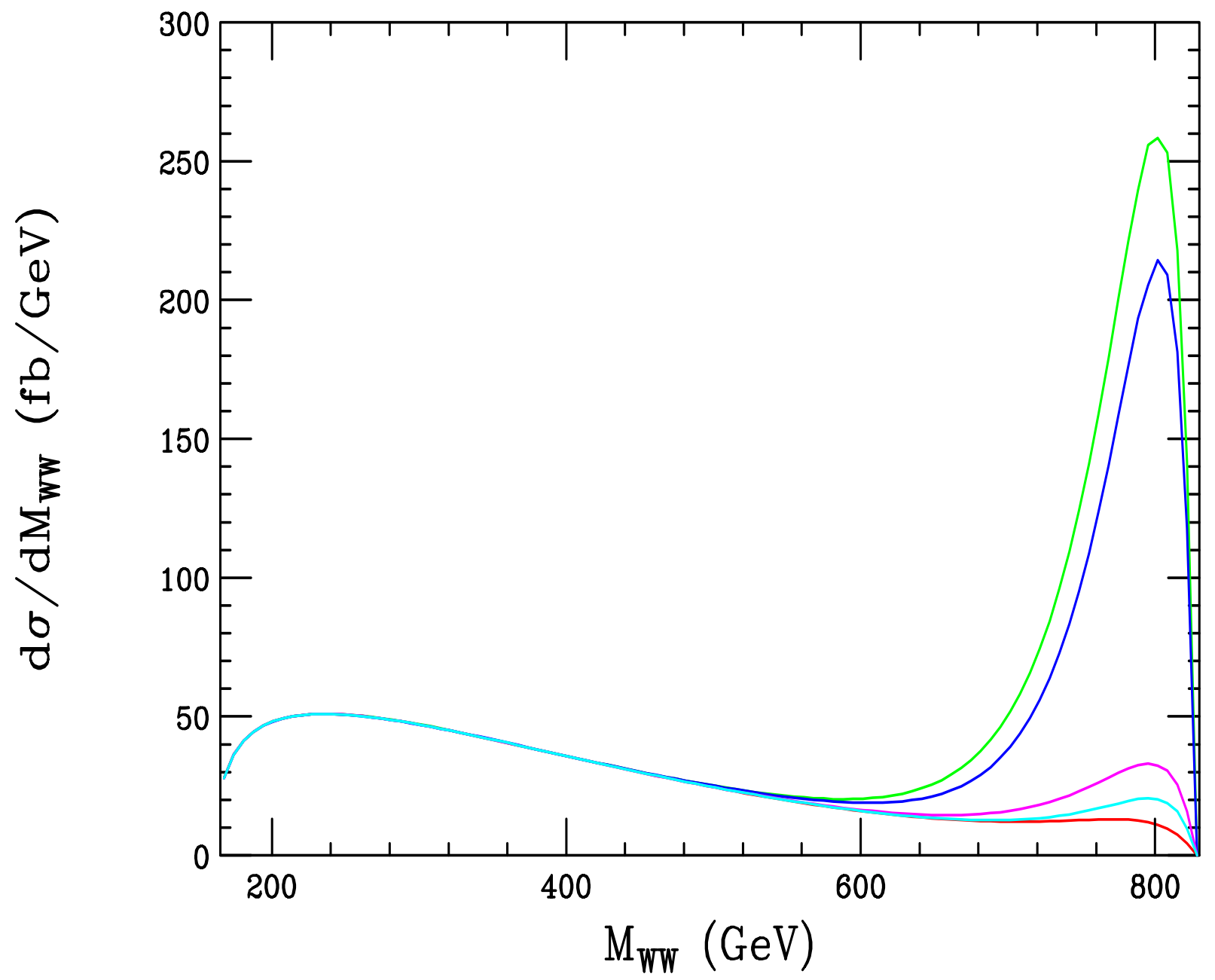

Figure 5: $W W$ mass distribution for $\gamma \gamma \rightarrow W^{+} W^{-}$at a $1 \mathrm{TeV} e^{+} e^{-}$collider for the initial polarization of $(-++-)$ in the $\mathrm{SM}(\mathrm{red})$, the case of graviton exchange with $M_{s}=2.5 \mathrm{TeV}$ for both values of $\lambda$ (green and blue, respectively) and for the corresponding scenario with $M_{s}=3.5 \mathrm{TeV}$ (magenta and cyan, respectively). In all cases a cut of $|z|<0.8$ has been imposed. 
from distinct final state polarization states. Such a general class of asymmetries involving differences in initial state photon helicities form the basic ingredients of the Drell-HearnGerasimov Sum Rule(DHG)[10] which has be applied, e.g., in the search for anomalous couplings between photons and $W, Z$ 's[11]. In order to prove the usefulness of these asymmetries let us first examine their angular dependencies in both the SM and when K-K towers of gravitons[12] are exchanged; this is shown in Fig.6 for a $1 \mathrm{TeV}$ collider. Note that as $z \rightarrow \pm 1$ the SM dominates due to the large magnitude of the $u$ - and $t$-channel poles. Away from the poles the three asymmetries all show a significant sensitivity to the K-K tower of graviton exchange. Although these asymmetries are not very big the large statistics of the data samples obtainable for this channel indicate that they will be very well determined since many systematic errors will also cancel in forming the cross section ratios.

As is well known[11] in order to satisfy the DHG sum rule the integrated asymmetry must pass through a zero (by the mean value theorem) for some value of $\hat{s}$. The exact position of the zero has been shown to be sensitive to new physics and to any applied kinematic cuts. What happens in the case where the SM is augmented by an exchange of a K-K tower of gravitons and how does $A_{p o l}$ vary with $M_{W W}$ ? Fig.7 address these question directly for all three independent asymmetries after employing the $|z|<0.8$ cut. The position of the zero is seen to be the same in all three cases and quite insensitive to even low values of $M_{s} \sim 2.5 \mathrm{TeV}$. This is due to the rather unfortunate fact that the zero occurs in the low $M_{W W}$ region where contributions from finite $M_{s}$ are very difficult to observe. The behaviour of $A_{p o l}$ at larger values of $M_{W W}$ is, however, very sensitive to graviton exchange indicating a strong modification of the Sum Rule integral itself. Since the K-K graviton tower exchange is represented by an effective dimension-eight operator it can be shown for all initial state helicities that the integral in the Sum Rule diverges. This should be expected since the new operator is non-renormalizable. 


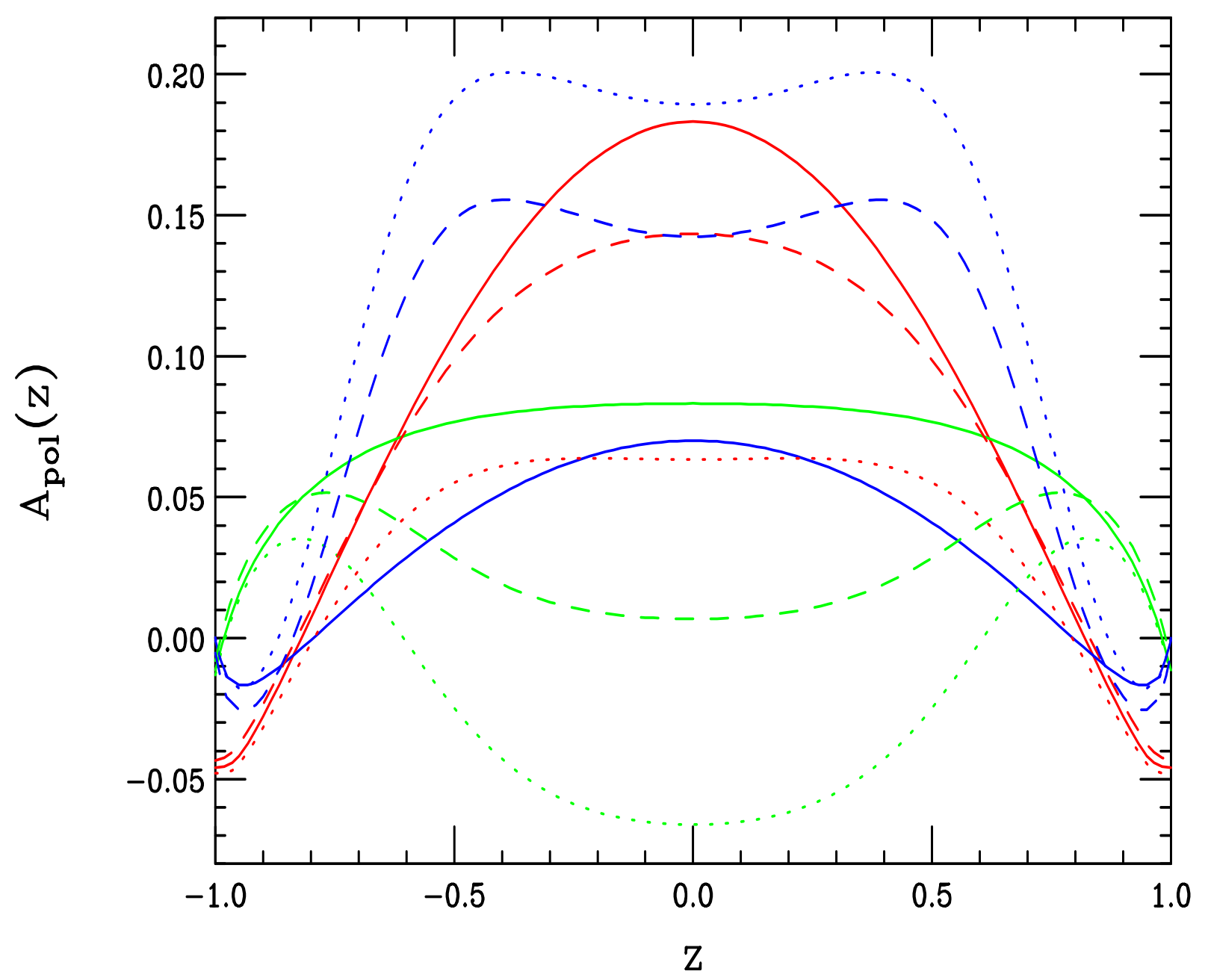

Figure 6: Differential polarization asymmetries for $\gamma \gamma \rightarrow W^{+} W^{-}$at a $1 \mathrm{TeV} e^{+} e^{-}$collider for the $\mathrm{SM}$ (solid) as well with graviton tower exchange with $M_{s}=2.5 \mathrm{TeV}$ with $\lambda= \pm 1$ (the dotted and dashed curves). We label the three cases shown by the first entry in the numerator in the definition of $A_{p o l}$. Red represents an initial polarization of $(++++)$, green is for the choice $(+++-)$ and blue is for the case $(-++-)$. 


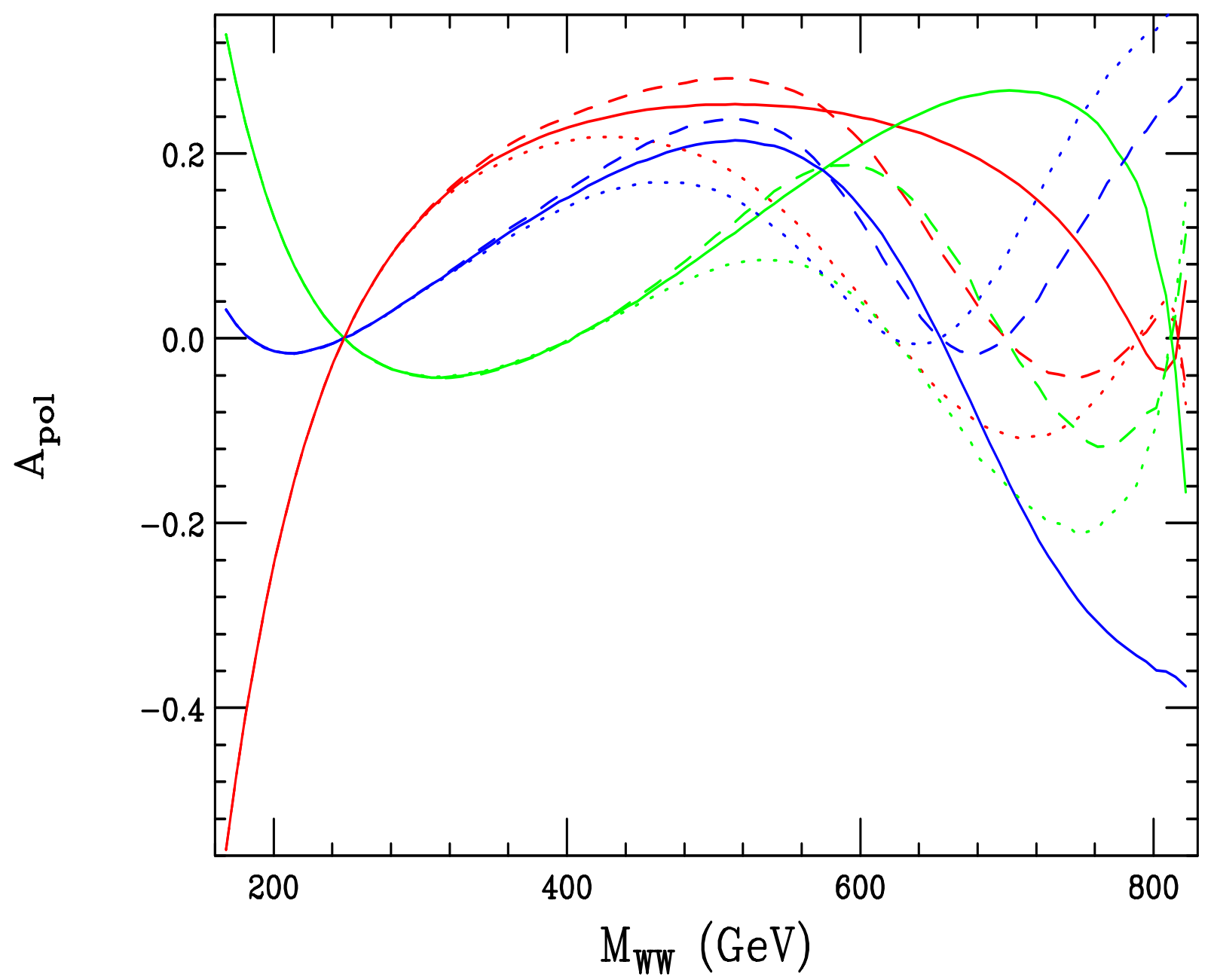

Figure 7: Integrated polarization asymmetries for $\gamma \gamma \rightarrow W^{+} W^{-}$at a $1 \mathrm{TeV} e^{+} e^{-}$collider as functions of the $W W$ invariant mass. The labels for the various curves are as in the previous figure and a cut of $|z|<0.8$ has been applied. 
It is clear from the discussion above that there are a large number of observables that can be combined into a global fit to probe very high values of $M_{s}$ in comparison to the collider energy. It should be noted however that due to the large statistics available the eventually determined discovery reach for $M_{s}$ using the $\gamma \gamma \rightarrow W^{+} W^{-}$process will strongly depend on the size and variety of the experimental systematic errors.

\section{$3.2 \gamma \gamma \rightarrow Z Z$}

The process $\gamma \gamma \rightarrow Z Z$ does not occur at the tree level in the SM or MSSM. At the one loop level in the SM the dominant contribution[13] arises from $W$ and fermion box diagrams and triangle graphs with s-channel Higgs boson exchange. (In SUSY models, additional contributions arise due to sfermion and gaugino loops as well as the other Higgs exchanges.) The $W$ bosons loops have been shown to be the dominant contribution with the fermions interfering destructively. This would seem to naively imply that this channel is particularly suitable for looking for new physics effects since the SM and MSSM rates will be so small due to the loop suppression. The SM cross section (which peaks in the forward and backward directions), after a cut of $|z|<0.8$, is found to be $\sim 80 \mathrm{fb}$ and almost purely transverse away from Higgs boson resonance peaks. The size of this cross section being only $\sim 10^{-3}$ of that for $W^{+} W^{-}$will make the $Z Z$ final state difficult to find. Since it is not anticipated that the $W$ and $Z$ masses will be very well separated in the jet-jet channel at this level of rejection, we must demand that at least one of the $Z$ 's decay leptonically reducing the effective number of useful $Z$ by a factor $\simeq 10$. (We can regain some reasonable fraction of this suppression depending on the practicality of the $q \bar{q} \nu \bar{\nu}$ final state. While this state is useful for constructing angular distributions assuming a very hermetic detector it cannot be used, e.g., to probe the polarization fractions of both of the $Z$ 's. The addition of this final 
state for other analyses would yield an efficiency of $\sim 50 \%$ instead of $\sim 10 \%$.) A luminosity of $100 \mathrm{fb}^{-1}$ thus yields a sample of only about $800(4000) Z$ pairs after these simple cuts and efficiencies are employed(assuming the $q \bar{q} \nu \bar{\nu}$ final states are included).

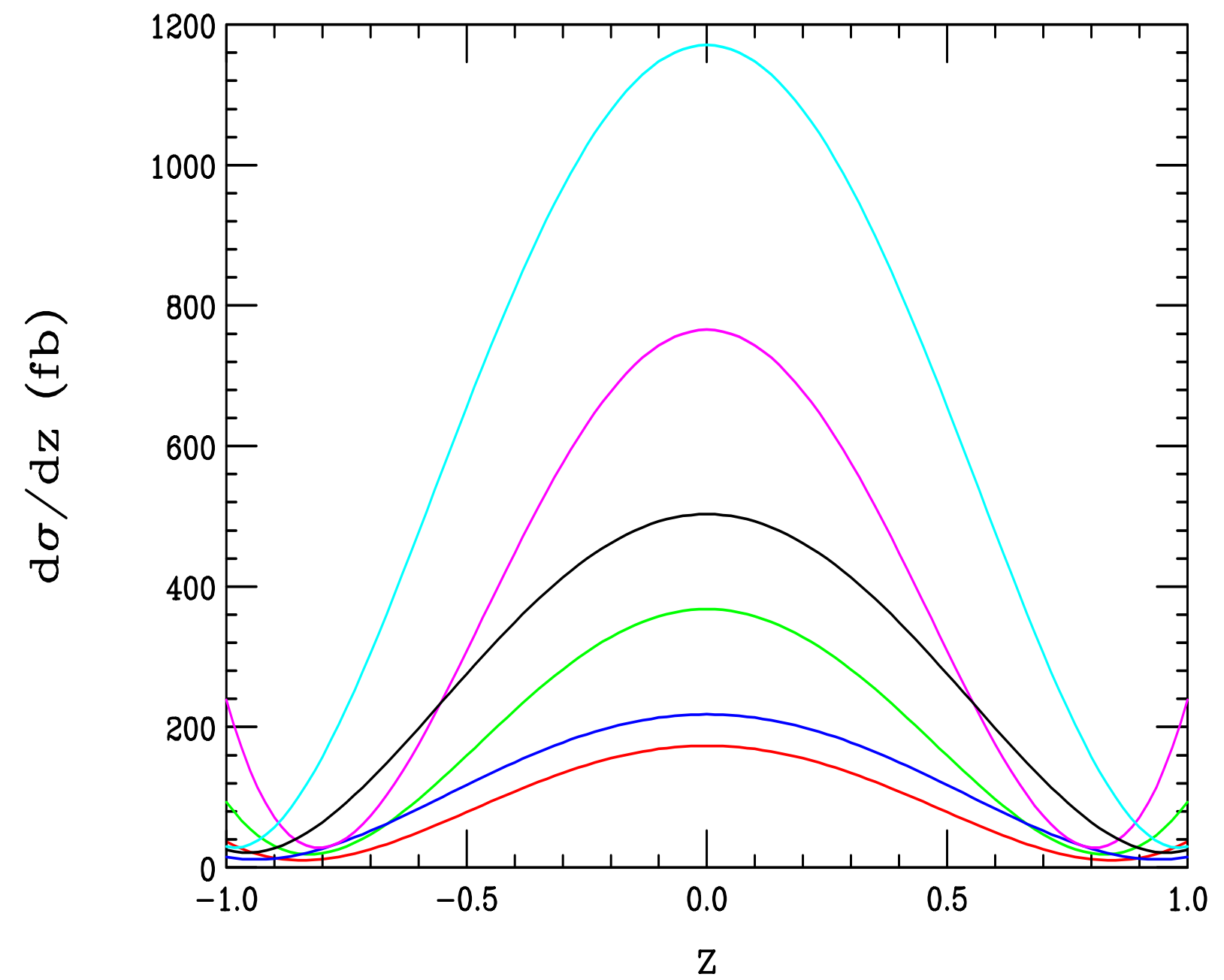

Figure 8: Differential cross section for $\gamma \gamma \rightarrow Z Z$ at a $1 \mathrm{TeV} e^{+} e^{-}$collider due to the exchange of a K-K tower of gravitons assuming $M_{s}=3 \mathrm{TeV}$. From top to bottom in the center of the figure the initial state helicities are $(-++-),(+-+-),(+---),(+++-),(++--)$, $(++++)$.

In the case of the ADD scenario the tree level K-K graviton tower contribution is now also present and is given by Eq.(2). Neglecting the loop-order SM contributions for the moment we obtain the resulting polarization-dependent differential cross sections shown in 
Fig. 8 where we have assumed $\sqrt{s}=1 \mathrm{TeV}$ and $M_{s}=3 \mathrm{TeV}$ for purposes of demonstration. Note that since this is the pure K-K graviton tower term there is no dependence here on the sign of $\lambda$. This cross section is found to scale with $s$ and $M_{s}$ as $\sim s^{3} / M_{s}^{8}$ and in contrast to the SM case is observed to peak at $90^{\circ}$. A short analysis along the lines of that performed above shows that essentially all of the $Z$ 's in the final state are completely longitudinal with the $L L$ fraction being $\sim 99 \%$ for the six possible initial state polarizations. Applying the same cuts, efficiencies and luminosities as above we find that for $M_{s}=4 \mathrm{TeV}$ this pure graviton contribution will only lead to 11(55) additional events beyond SM expectations which is not a huge excess. However, since the graviton tower cross section rises as $\hat{s}^{3}$ while the luminosities are falling off slowly, making a cut on the $Z Z$ invariant mass, $M_{Z Z}>550$ $\mathrm{GeV}$, one finds that essentially all of the K-K-induced events lie above this cut (for the assumed value of the integrated luminosity) and would appear similar to a resonance-almost a broad Higgs-like[13] bump. However, it would be doubtful that such an excess would be observed if $M_{s}>5 \mathrm{TeV}$ due to the $\sim M_{s}^{-8}$ scaling behaviour of the cross section unless significantly higher luminosities were available and systematic errors were very much under control. In addition, the use of the longitudinal polarization fraction would not seem to gain us any further reach.

Although a detailed study of the loop-induced SM-graviton tower exchange interference terms have not yet been performed it is difficult to see how the search reach in this channel can exceed $\simeq 5 \mathrm{TeV}$ given the small magnitudes of the cross sections involved. Thus the anticipated overall reach for the $\gamma \gamma \rightarrow Z Z$ process is reasonably similar to that found for $\gamma \gamma \rightarrow \gamma \gamma[5]$ 


\section{Summary and Conclusions}

$\gamma \gamma \rightarrow W^{+} W^{-}, Z Z$ offer new channels in which to search for the influence of graviton tower exchange, naively, each with their own individual strengths and weaknesses. We have found that

- The SM cross section for $\gamma \gamma \rightarrow W^{+} W^{-}$is very large even after reasonable angular cuts are applied providing enormous statistics to look for new physics. This large cross section leads to an amplification of the size of the SM-graviton interference terms. The

final state is quite clean there being little backgrounds due to rates alone and $90 \%$ of the decay products are useable for analyses. The differential cross section as well as the polarization of the $W$ 's in the final state were found to be quite sensitive to graviton exchanges especially for certain initial state electron and laser polarizations. The $W$-pair invariant mass distribution and the analogs of the Drell-Hearn-Gerasimov polarization asymmetries were also shown to be able to probe large values of $M_{s}$. Fitting the cross section and final state polarizations, after cuts, efficiencies and systematic errors were included was shown to lead to search reaches $\sim 11 \sqrt{s}$, the largest of any of the graviton exchange processes so far examined. Detailed simulations of this channel should be performed which include the other observables, radiative corrections and detector effects to verify or improve upon this reach estimate.

- $\gamma \gamma \rightarrow Z Z$ would also appear to be an excellent process to probe for large values of $M_{s}$ since it only occurs at the loop level in the SM and MSSM. However both the SM and K-K tower cross sections are quite small for $M_{s}>4 \mathrm{TeV}$ even if the most advantageous initial state polarization choice, $(-++-)$, is assumed. Some assistance is gained by the fact that almost all of the $Z Z$ events will lie above a cut of $M_{Z Z}>550-600 \mathrm{GeV}$ and that they are almost purely longitudinally polarized. It seems unlikely, however, 
that the search reach in this channel can much exceed $5 \mathrm{TeV}$ unless very large data samples become available.

The physics on large extra dimensions is quite exciting and may reveal itself at collider experiments in the not too far distant future.

\section{Acknowledgements}

The author would like to thank J.L. Hewett, N. Arkani-Hamed, J. Wells, T. Han, J. Lykken, M. Schmaltz and H. Davoudiasl for multi-dimensional discussion related to this work. He would also like to thank M. Berger, D. Dicus and G.J. Gounaris for discussions related to the SM helicity amplitudes for $\gamma \gamma \rightarrow Z Z$. 


\section{References}

[1] N. Arkani-Hamed, S. Dimopoulos and G. Dvali, Phys. Lett. B429, 263 (1998) and Phys. Rev. D59, 086004 (1999); I. Antoniadis, N. Arkani-Hamed, S. Dimopoulos and G. Dvali, Phys. Lett. B436, 257 (1998;)N. Arkani-Hamed, S. Dimopoulos and J. MarchRussell, hep-th/9809124; P.C. Argyres, S. Dimopoulos and J. March-Russell, Phys. Lett. B441, 96 (1998); Z. Berezhiani and G. Dvali, hep-ph/9811378; N. Arkani-Hamed and S. Dimopoulos, hep-ph/9811353; Z. Kakushadze, hep-th/9811193 and hep-th/9812163;

N. Arkani-Hamed et al., hep-ph/9811448; G. Dvali and S.-H.H. Tye, hep-ph/9812483. See also, G. Shiu and S.-H. H. Tye, Phys. Rev. D58, 106007 (1998); Z. Kakushadze and S.-H. H. Tye, hep-th/9809147; I. Antoniadis, Phys. Lett. B246, 377 (1990); J. Lykken, Phys. Rev. D54, 3693 (1996); E. Witten, Nucl. Phys. B471, 135 (1996); P. Horava and E. Witten, Nucl. Phys. B460, 506 (1996) and Nucl. Phys. B475, 94 (1996); K.R. Dienes, E. Dudas and T. Gherghetta, Phys. Lett. B436, 55 (1998), Nucl. Phys. B538, 47 (1999)and hep-ph/9807522.

[2] J.C. Long, H.W. Chan and J.C. Price, hep-ph/9805217.

[3] S. Cullen and M. Perelstein, hep-ph/9903422 and references therein.

[4] L.J. Hall and D. Smith, hep-ph/9904267.

[5] G.F. Guidice, R. Rattazzi and J.D. Wells, Nucl. Phys. B544, 3 (1999); T. Han, J.D. Lykken and R.J. Zhang, Phys. Rev. D59, 105006 (1999); J.L. Hewett, hep-ph/9811356; E.A. Mirabelli, M. Perelstein and M.E. Peskin, Phys. Rev. Lett. 82, 2236 (1999); P. Mathews, S. Raychaudhuri and K. Sridhar, hep-ph/9811501, Phys. Lett. B450, 343 (1999), and hep-ph/9904232; S. Nussinov and R.E. Shrock, Phys. Rev. D59, 105002 (1999); T.G. Rizzo, hep-ph/9901209, hep-ph/9902273 and hep-ph/9903475; K. Agashe 
and N.G. Deshpande, hep-ph/9902263; M.L. Graesser, hep-ph/9902310; K.Cheung and W.-Y. Keung, hep-ph/9903294; N. Arkani-Hamed and M. Schmaltz, hep-ph/9903417; A.K. Gupta, N.K. Mondal and S. Raychaudhuri, hep-ph/9904234; C. Balazs, H.-J. He, W. Repko, C.P. Yuan and D. Dicus, hep-ph/9904220; D. Atwood, S. Bar-Shalom and A. Soni, hep-ph/9903538; L. Hall and C. Kolda, hep-ph/9904236; G. Shiu, R. Shrock and S.-H. Tye, hep-ph/9904262; the process $\gamma \gamma \rightarrow \gamma \gamma$ has been analyzed by K. Cheung, hep-ph/9904266; H. Davoudiasl, SLAC-PUB-8121 .

[6] For a recent review of $\gamma \gamma$ colliders, photon distributions and original references, see $\mathrm{V}$. Telnov, hep-ex/9810019. For more details, see I.F. Ginzburg et al., Nuc. Inst. Meth. 205, 47 (1983), Nuc. Inst. Meth. 219, 5 (1984), Nuc. Inst. Meth. A294, 2 (1990) and Nuc. Inst. Meth. A355, 3 (1995); V.I. Telnov, Nuc. Inst. Meth. A294, 72 (1990); D.L. Bordon, D.A. Bauer and D.O. Caldwell, SLAC-PUB-5715 (1992).

[7] This was pointed out by S. Chakrabarti et al., Phys. Lett. B434, 347 (1998).

[8] The complete SM helicity amplitudes are given in G. Belanger and F. Boudjema, Phys. Lett. B288, 210 (1992); E. Yehudai, Phys. Rev. D44, 3434 (1991).

[9] The full one-loop electroweak corrections for $\gamma \gamma \rightarrow W^{+} W^{-}$are found in great detail in G. Jikia, Nucl. Phys. B494, 19 (1997).

[10] S. D. Drell and A. C. Hearn, Phys. Rev. Lett. 16, 908 (1966); S. Gerasimov, Yad. Fiz. 2, 598 (1965) [Sov. J. Nucl. Phys. 2, 430 (1966)]; L. I. Lapidus and Chou Kuang-Chao, J. Exptl. Theoretical Physics 41, 1545 (1961) [Sov. Phys. JETP 14, 1102 (1962)]; M. Hosada and K. Yamamoto, Prog. Theor. Phys. 36, 426 (1966). For a recent review of the empirical tests of the DHG sum rule see B. L. Ioffe, preprint ITEP-61 (1994); D. Drechsel, University of Mainz preprint, 1994. J.L. Hewett and J.D. Wells, Phys. Rev. 
D55, 5549 (1997). An analysis of the relationship of anomalous couplings to internal structure for spin-1 bound states is discussed in F. Schlumpf and S.J. Brodsky, Phys. Lett. B360, 1 (1995); S. J. Brodsky and I. Schmidt, Phys. Lett. B351, 344 (1995).

[11] S.J. Brodsky, T.G. Rizzo, and I. Schmidt, Phys. Rev. D52, 4929 (1995); T.G. Rizzo, Phys. Rev. D54, 3057 (1996).

[12] The use of the DHG sum rule in the presence of graviton emission has recently been discussed by H. Goldberg, hep-ph/9904318.

[13] The loop induced amplitudes and cross section in the SM have been computed in G. Jikia, Nucl. Phys. B405, 24 (1993); E.W.N. Glover and J.J. van der Bij, Nucl. Phys. B318, 106 (1989) and Nucl. Phys. B321, 561 (1989). See also D.A. Dicus and C. Kao, Phys. Rev. D49, 1265 (1994) and M.S. Berger, Phys. Rev. D48, 5121 (1993). 